\title{
Hepatoportal sclerosis
}

INSERM

\section{Source}

INSERM. (1999). Orphanet: an online rare disease and orphan drug data base.

Hepatoportal sclerosis. ORPHA:64743

Hepatoportal sclerosis (HPS) is a rare disorder characterized by sclerosis of the intrahepatic portal veins, non-cirrhotic portal hypertension, asymptomatic splenomegaly and recurrent variceal bleeding. 